\title{
EDITORIAL
}

\section{The Clumsy Child: Where Are We and Where Are We Going?}

In June 2002, a meeting was held in Groningen, the Netherlands on 'The Clumsy Child'. Here neuroscientists who presented recent findings on the organization and development of motor control met clinicians involved in the management and care of children with clumsy motor behavior. The present special issue of Neural Plasticity contains the main contributions of this meeting, as well as an edited version of the lively discussion held at the end of the meeting.

The issue begins with the paper of Henderson and Henderson who eloquently describe all the difficulties that researchers run into when dealing with children with clumsy motor behavior. To avoid difficulties with terminology, they plea for the general use of the DSM-IV term Developmental Coordination Disorder (DCD, American Psychiatric Association, 1994) to describe this group of children. The authors also discuss the hurdles encountered in diagnosing DCD and the comorbid signs and symptoms of in the heterogeneous population of children with clumsy motor behavior.

Next is a theoretical paper by Hopkins, who points out that the development of action can be considered as consisting of changes in the taskspecific couplings between perception, movement and posture. He argues that this perception-action approach can provide a much-needed basis from which attempts can be made at theoretically unifying the constituents of the clinical continuum of normal motor behavior to motor clumsiness. In the following paper, Sigmundsson discusses the issue of perceptual deficits in clumsy children. $\mathrm{He}$ argues that in particular asymmetries in the processing of information and motor control might result in motor coordination problems.

The following two papers address the etiology of clumsy motor behavior. Hadders-Algra proposes that in only a minority of children can clumsiness be attributed to a lesion of the brain, particularly to damage of the fiber systems connecting the various supraspinal regions. She argues that in most clumsy children, clumsiness represents the lower scale of the normal distribution of motor function. Such motor performance probably reflects non-pathological-brain function due to factors like genetic constitution and stress during early life. Mercuri and Barnett report on the motor consequences of neonatal encephalopathy in full-term infants and conclude that especially infants who on MRI show signs of severe basal ganglia and internal capsule involvement are at risk for the development of motor impairment, such as cerebral palsy and clumsy motor behavior. The next paper by Gillberg and Kadesjö addresses comorbidity in children with DCD. Psychiatric disorders like attention deficit hyperactivity disorder (ADHD) and autistic spectrum disorders are much more common in children with DCD than in those without this developmental motor disorder, a notion having clinical implications.

The following section contains seven papers reviewing the role of a specific part of the nervous system in motor control. Kernell begins with a scholarly survey on how motoneurones and motor units are used for the gradation of muscle force during motor behavior. The next two papers deal with the effect of a lesion of corticospinal tract. Whishaw and his colleagues studied the effect of complete and partial lesions of the pyramidal tract in adult rats. They found not only that complete 
lesions result in clear motor deficits but also that partial lesions induce motor abnormalities, albeit minor ones that can be detected only with the help of a detailed analysis of the performance of reaching behavior. The paper by Eyre presents an overview on the development and plasticity of the corticospinal tract in man. She points out that the corticospinal system develops from a rather diffuse system into a specific contralateral system and the reorganizational processes induced by unilateral and bilateral cortical lesions differ. The next two papers discuss the basal ganglia in motor control. Groenewegen succinctly describes the anatomy of the basal ganglia, a neural system that supports a wide range of sensorimotor, cognitive, and emotional-motivational brain functions. He points out that a major role of the basal ganglia is the learning and selection of the most appropriate motor or behavioral programs. The second paper on basal ganglia is by Keitz, Martin-Soelch and Leenders who review the literature on dopaminergic circuitries in reward processing and speculate upon the role of reward-processing neural systems in motor behavior. Two papers on the role of the cerebellum in the development of motor behavior conclude this section. Gramsbergen presents a review on cerebellar neuroanatomy, neurophysiology, and cerebellar development. $\mathrm{He}$ stresses the relative late maturation of cerebellar structure and points to the possible consequences of the timing of cerebellar development for normal and-in particular-for abnormal development induced by perinatal adversities like fetal growth retardation. The second paper is by Ivry. He discusses the association of cerebellar anomalies and developmental disorders like ADHA, dyslexia, and autism, and the putative causational links between cerebellar anomalies and these various disorders. In addition, he suggests that the major role of the cerebellum might be the control of timing of neural events, a role that is restricted not only to motor events but also might hold true for perceptual tasks.

The final contribution is by Schoemaker et al. They deal with the important issue of clinical management of clumsy motor behavior of childhood. The authors point out that traditional physiotherapeutic intervention, following the principles of neurodevelopmental or sensory integration, is only moderately successful. Novel forms of intervention using a task-oriented approach seem to be more promising for the remediation of clumsiness.

The papers in this special issue reveal that our understanding of motor development in humans and the pathophysiology of motor clumsiness in childhood is only in its infancy. This implies not only that our current knowledge is relatively limited but also that it has a vigorous potential to grow, as illustrated by some promising perspectives opened in the recent past. For example, the introduction of physiological measurement techniques that can be applied in children, including recording the EMG of multiple muscles during everyday childhood motor tasks and functional imaging of the brain, will help us to explain the neural basis of clumsiness. This, in turn, will facilitate the development of effective intervention strategies. The possibilities are promising and exciting and form the feeding fuel on the long route to the full understanding of clumsy motor behavior and its management. A route that will consist of multidisciplinary research in fields ranging from molecular neurobiology to neurorehabilitation, with a major role for studies using the techniques of systems physiology.

Mijna Hadders-Algra and Albert Gramsbergen

\section{Reference}

American Psychiatric Association. 1994. Diagnostic and Statistical Manual of Mental Disorders, $4^{\text {th }}$ ed. Washing, DC, USA: APA. 

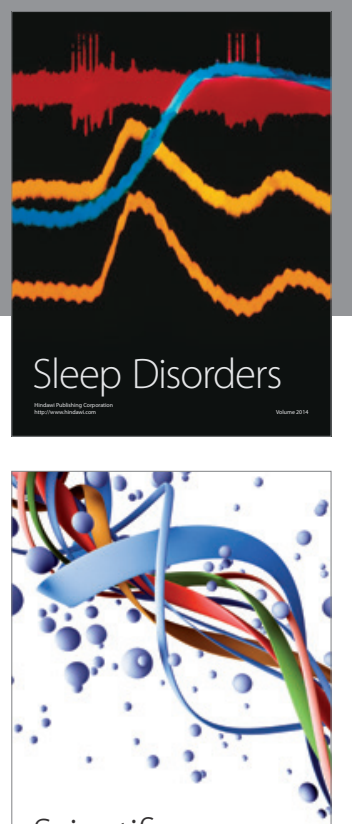

Scientifica
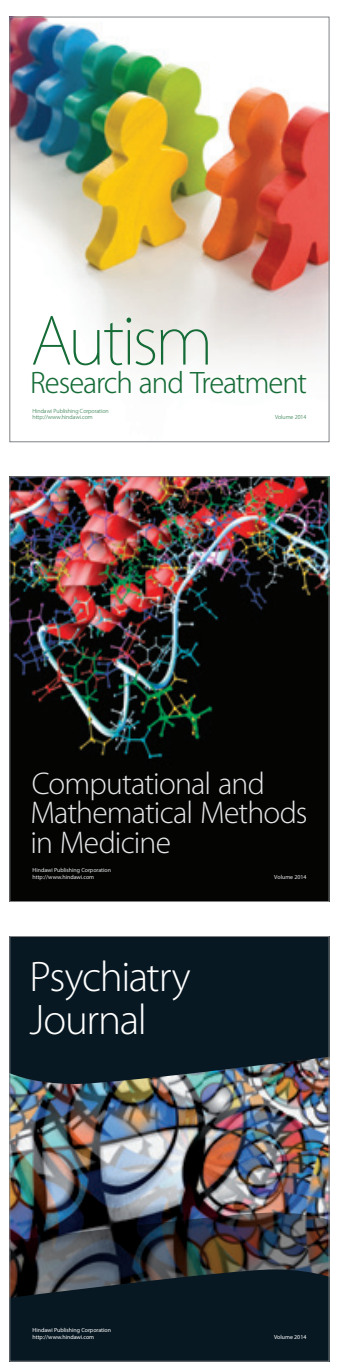
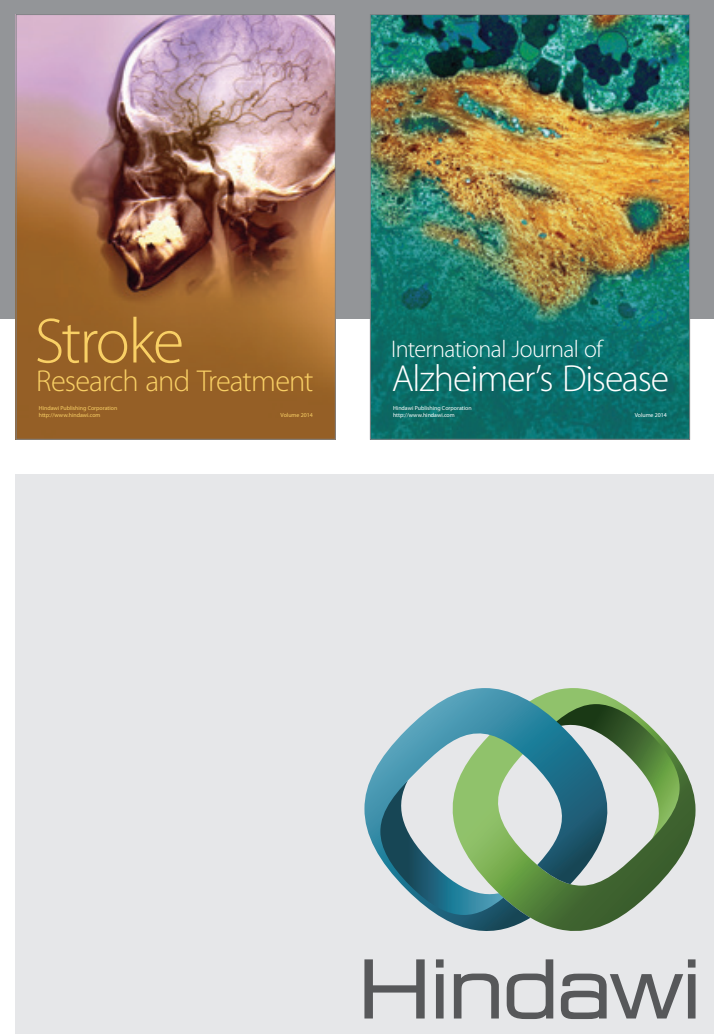

Submit your manuscripts at

http://www.hindawi.com
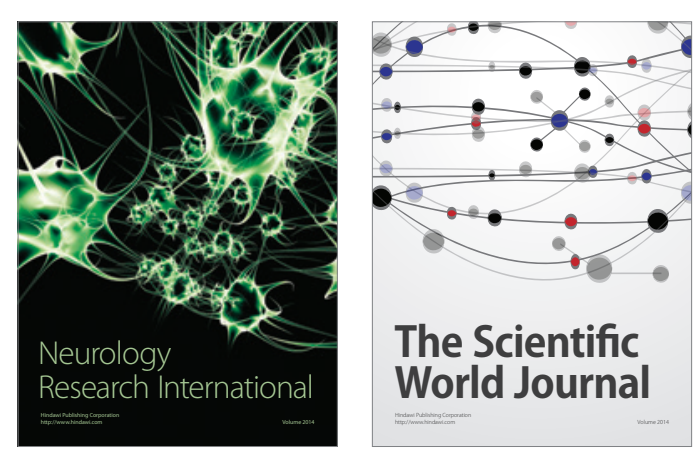

The Scientific World Journal

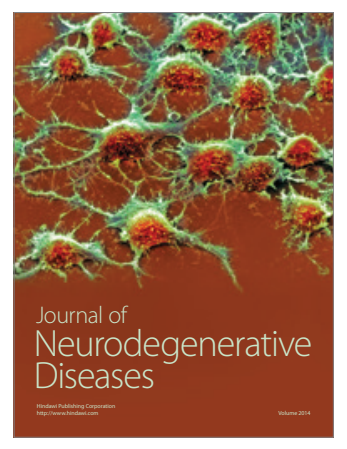

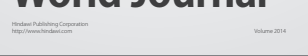

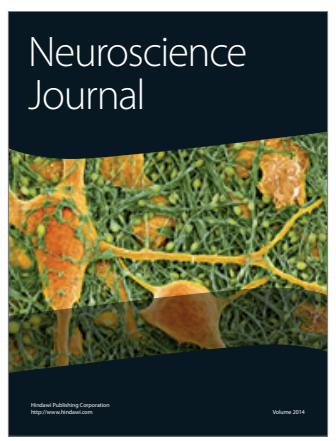

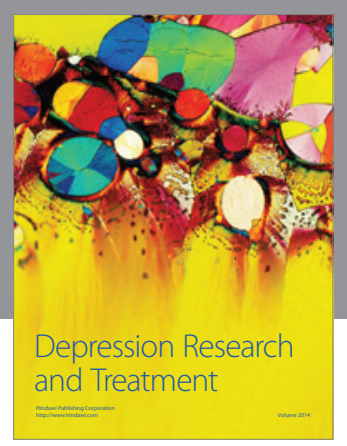
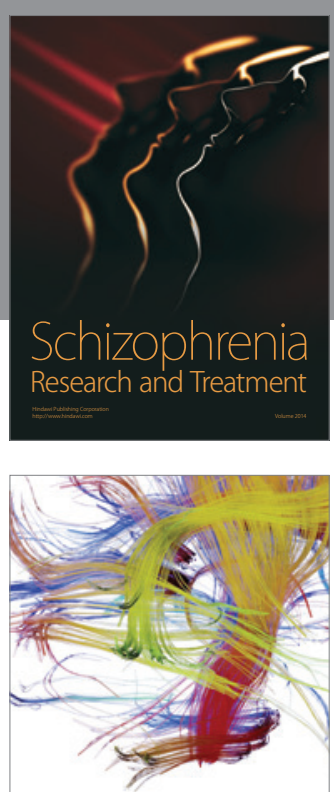

Brain Science

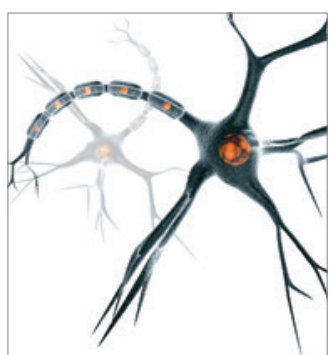

Neural Plasticity
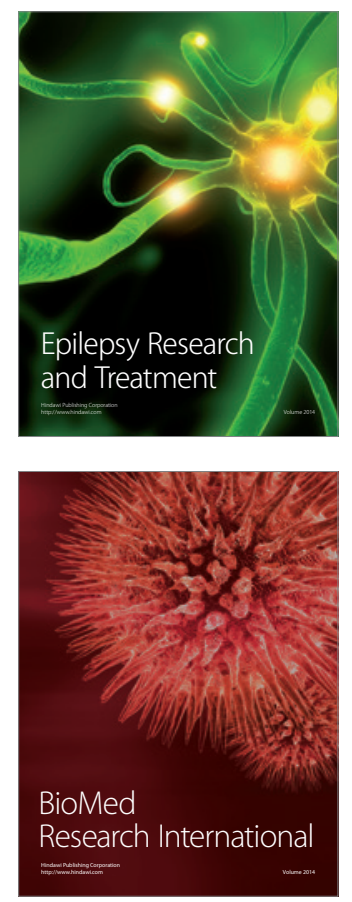

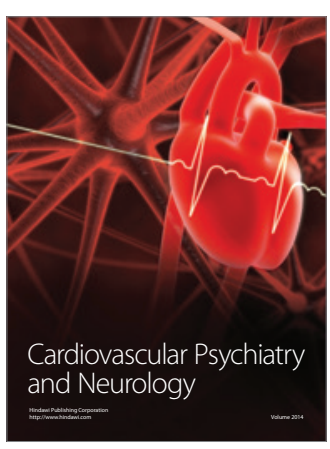

Parkinson's

Disease
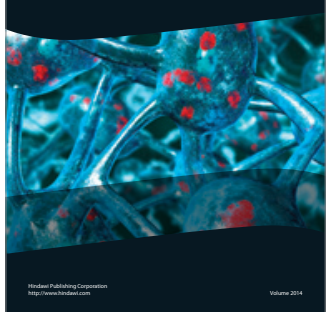